\title{
Biorremediación de suelo contaminado con 75000 ppm de aceite residual automotriz por bioestimulación y fitorremediación con Sorghum vulgare y Bacillus cereus y/ o Burkholderia cepacia
}

\section{Biorremediation of soil polluted by 75000 ppm of waste motor oil applying biostimulation and phytoremediation with Sorghum vulgare and Bacillus cereus or Burkholderia cepacia}

Balderas-León Iván ${ }^{1}$, Sánchez-Yáñez Juan Manuel ${ }^{1 *}$

\begin{tabular}{l}
\hline Datos del Artículo \\
\hline ${ }^{1}$ Microbiología Ambiental, Instituto \\
de Investigaciones Químico Bioló- \\
gicas, Ed-B3. \\
*Dirección de contacto: \\
Juan Manuel Sánchez-Yáñez: \\
Universidad Michoacana de San \\
Nicolás de Hidalgo, Francisco J. \\
Mujica S/N, Col. Felicitas del Rio \\
C.P. 58000, Morelia, Mich, México. \\
E-mail address: \\
syanez@umich.mx
\end{tabular}

\section{Palabras clave:}

Equilibrio mineral, fisiología vegetal, mineralización.

\section{Resumen}

En el suelo contaminado en alta concentración de aceite residual automotriz (ARA) causa pérdida de su fertilidad. Una solución es la biorremediación (BR) por doble y secuencial bioestimulación (BS) y posterior fitorremediación (FR) con Sorghum vulgare y Bacillus cereus/Burkholderia cepacia o bacterias promotoras de crecimiento vegetal (BPCV) para reducir el ARA remanente. Los objetivos de este trabajo fueron: a) biorremediar un suelo contaminado con 75000 ppm de ARA por doble y secuencial bioestimulación, y posterior b) fitorremediación con $S$. vulgare y las BPCV. El suelo contaminado con ARA se biorremedio por BS primero con solución mineral (SM), una segunda BS con Phaseolus vulgaris y BPCV incorporada como abono verde (AV), después por fitorremediación con $S$. vulgare y las BPCV para minimizar el ARA. Los resultados indicaron que la biorremediación del suelo por doble y secuencial BS: con SM el ARA decreció a $32500 \mathrm{ppm} / 30$ días y con P. vuglaris; lo disminuyo hasta $10100 \mathrm{ppm} / 90$ días. Su fitorremediación para minimizar el ARA remanente con $S$. vulgare y BPCV a floración lo redujo de 2500 ppm a 800 ppm. Ello apoya que biorestaurar un suelo impactado con elevada concentración de ARA; la mejor es la integración de la BR/FR, que su aplicación por separado.

(C) 2015. Journal of the Selva Andina Research Society. Bolivia. Todos los derechos reservados.

\section{Abstract}

\section{J. Selva Andina Res. Soc.} 2015; 6(1):23-32.

\section{Historial del artículo.}

Recibido, septiembre, 2014 Devuelto, noviembre 2014

Aceptado, febrero, 2015

Disponible en línea, febrero, 2015

Editado por:

Selva Andina

Research Society

\section{Key words:}

Mineralization, mineral balance, plant physiology.
Waste motor oil (WMO) pollutes soil and causing lost soil fertility. An alternative to solve this problem its bioremediation (BR) by double and following biostimulation (BS) with mineral solution (MS) and a legume as green manure (GM) then using phytoremediation (PR) with growth promoting vegetal bacteria (GPVB) like Bacillus cereus and Burkholderia cepacia to minimize remaining WMO. The aims of this research were: a) bioremediation of polluted soil by $75000 \mathrm{ppm}$ of WMO by biostimulation and then b) Its phytoremediation for remaining WMO by Sorghum vulgare inoculated with B. cereus and B. cepacia. Soil polluted by high concentration WMO was biostimulated with MS, and then Phaseolus vulgaris treated by GPVB was incorporated as GM, finally to apply PR to eliminate WMO with $S$. vulgare with GPVB. Results indicate that soil bioremediated by biostimulation with MS, WMO decreased at $32500 \mathrm{ppm} / 30$ days, and then with GM, WMO was reduced at $10100 \mathrm{ppm}$ after/90 days. Finally, to apply phytoremediation using $S$. vulgare and GPVB at flowering, WMO was reduced from $2500 \mathrm{ppm}$ to $800 \mathrm{ppm}$. For recovering soil impacted by high concentration WMO to apply both techniques double and following BS and PR are the best option than each technique separately. 


\section{Introducción}

En suelo el derrame en exceso en concentración superior a $35000 \mathrm{ppm}$ de aceite residual automotriz (ARA) una mezcla de hidrocarburos (HC) alifáticos, aromáticos y algunos metales pesados (Ogbo et al. 2009, Maldonado-Chávez et al. 2010) impide el intercambio gaseoso e inhibe la mineralización de materia orgánica relacionada con sus ciclos biogeoquímicos que sustenta la vida y afecta drásticamente la producción agrícola. En ese caso la alternativa de solución es aplicar un método químico dadas las mínimas posibilidades de éxito empleando únicamente ya sea la biorremediación (BR) o fitorremediación (FR) (Domínguez-Rosado et al. 2004, Zand et al. 2010). Cuando se ha intentado eliminar HC en este relativo elevado nivel de ARA, los resultados han sido negativos incluso cuando se ha empleado una sola estrategia de BR seguida por la fitorremediación (FR) sin inocular con BPCV. En suelo contaminado con 75000 ppm de ARA una alternativa ecológica que podría evitar el empleo de un agente químico costoso que deja residuos tóxicos al ambiente, podría ser una doble y secuencial BS: con la primera una solución mineral se induce a la microbiota aerobia heterotrófica autóctona de ese suelo, que inicie la mineralización de la fracción alifática del ARA; con la segunda BS la incorporación de Phaseolus vulgaris inoculada a nivel de semilla con un género de bacteria promotora de crecimiento vegetal (BPCV) del tipo Bacillus cereus un bacilo esporulado Gram positivo; benéfica tanto para gramíneas como leguminosas por la síntesis de sustancias promotoras de crecimiento vegetal (SPCV), al igual que Burkholderia cepacia un bacilo Gram negativo que también convierte exudados radicales en SPCV; ambos géneros reconocidos por su capacidad para oxidar algunos HC (Sánchez-Yáñez 2007) similares a los componentes del ARA. Normalmente las semillas de $P$. vulgaris se inoculan con un sola BPCV, una modalidad para mejorar su efecto positivo en plantas, es tratar esas semillas con dos o más géneros de BPCV, que aumenten la probabilidad de su acción benéfica en la planta en condición de estrés nutricional u ambiental, como la contaminación del suelo con una elevada concentración de ARA. Con base en lo anterior las semillas de $P$. vulgaris luego de inocularse con las BPCV, se sembraron, cuando la leguminosa alcanzo prefloración se incorporó al suelo en forma de abono verde (AV), para intentar reducir nuevamente la concentración de ARA (Sánchez-Yáñez 2007), necesaria para permitir la aplicación de la fitorremediación (FR) con Sorghum vulgare potenciada con las BPCV.

En el suelo esta doble BS contribuye a restaurar el equilibrio mineral e induce a su microbiota autóctona heterotrófica aerobia a oxidar los HC del ARA (Banks et al. 2003, Domínguez- Rosado $\&$ Pichtel 2004) y haga permisible el empleo de la FR con alguna con gramínea tolerante a la fitotoxicidad del ARA como el $S$. vulgare que podría mejorar su capacidad de fitodegradarlo al inoculado con BPCV y contribuyen la mineralización del ARA para acelerar la biorestauración del suelo (Basumatary et al. 2012, Osaigbovo et al. 2013).

En la literatura se reporta la BR y FR de suelo contaminado con ARA, aplicadas independiente y excepcionalmente de manera secuencial e integral cuando el nivel del ARA es relativamente bajo de entre 10000 y 30000 ppm. Así DomínguezRosado \& Pichtel (2004) reportaron la biores- 
tauración de suelo contaminado con 15000 ppm de ARA, por bioestimulación con una SM y su posterior FR para reducir el remanente con Helianthus annus y/o con Zea mays. Los resultados señalaron que el suelo con ARA bioestimulado con SM y su ulterior fitorremediación redujeron la concentración del ARA hasta 4950 ppm. En tanto Agamuthu et al. (2010) señalaron que la biorremediación de un suelo contaminado con 10000 y 25000 ppm de ARA por BS con una enmienda orgánica y después por fitorremediación para el restante con Jatropha curcas. Los resultados mostraron que el suelo con 10000 ppm de ARA; la integración y secuencial empleo de la BS y la FR lo redujo hasta 340 ppm. El mismo suelo con 25000 ppm de ARA, la acción en secuencia de ambas estrategias de BS/FR lo redujo a 2600 ppm en 180 días. Con base en estos antecedentes y la escasa la información sobre la estrategia integral y secuencia de la biorestauración de suelo contaminado con una elevada concentración de ARA. Los objetivos de esta investigación fueron i) la biorremediación de un suelo contaminado con 75000 ppm de ARA por doble y secuencial bioestimulación, ii) la fitorremediación para minimizar el ARA restante con Sorghum vulgare inoculado con Bacillus cereus y/o Burkholderia cepacia.

\section{Materiales y métodos}

Esta investigación se realizó en el invernadero del laboratorio de Microbiología Ambiental del Instituto de Investigaciones Químico Biológicas de la UMSNH. En ese invernadero las condiciones microclimáticas promedio fueron temperatura de $23.2^{\circ} \mathrm{C}$, luminosidad de 450 25 $\mu \mathrm{mol} \cdot \mathrm{m}^{-2} \cdot \mathrm{s}^{-1}$ y humedad relativa de $67 \%$. Para este ensayo se utilizó un suelo colectado de un sitio ubicado a los $19^{\circ} 39^{\prime} 27^{\prime}$ ' de latitud norte $100^{\circ} 19^{\prime} 59^{\prime}$ ' de longitud oeste, con una altitud de 1820 msnm de clima templado en un terreno agrícola denominado "La cajita" de la Tenencia Zapata del municipio de Morelia, Mich., México sobre el km 5 de la carretera Morelia-Pátzcuaro, Mich, México. Este suelo se clasifico como lateritico de textura franco arenosa, pobre en materia orgánica de $1.5 \%$ y $\mathrm{N}$ orgánico de 39 $\mathrm{Kg} / \mathrm{ha}$ y un $\mathrm{pH} 6.7$ ligeramente ácido. El suelo se solarizó a $70^{\circ} \mathrm{C} / 48 \mathrm{~h}$ para minimizar el problema de plagas y enfermedades, posteriormente se tamizó con una malla del No. 20 y contaminó con 75000 ppm de ARA proveniente de un taller mecánico automotriz diluido en agua con un detergente comercial (La Corona $\left.{ }^{\circledR}\right)$ al $1.0 \%(\mathrm{p} / \mathrm{v})$.

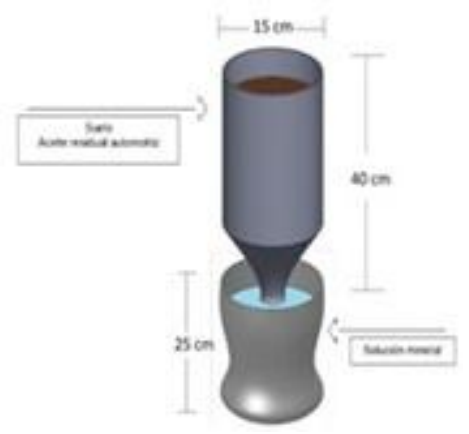

Figura 1 Diagrama de diseño de una jarra de Leonard

Luego un $1 \mathrm{~kg}$ suelo se colocó en el contenedor superior de cada jarra de Leonard, mientras que la SM o agua en el reservorio de la parte inferior, ambas partes se conectaron por una tira de algodón de aproximadamente de $20 \mathrm{~cm}$ de largo para permitir el movimiento de la SM o agua por capilaridad al suelo (Figura 1); según el tratamiento especificado en la Tabla 1 que describe el diseño experimental. El ensayo de 
biorremediación/fitorremediación del suelo impactado con alta concentración de ARA se dividió en dos fases de acuerdo con diseño experimental de bloques al azar con 5 tratamientos y 6 repeticiones señalados en la Tabla 1, en donde se describen 2 controles: el suelo sin contaminar con ARA humedecido solo con agua considerado como el control absoluto; sin biorremediar o fitorremediar; y el mismo suelo contaminado con ARA, humedecido con agua sin biorremediar o fitorremediar o control negativo y los tratamientos donde el suelo se empleó la doble y secuencial BS con SM y $P$. vulgaris como $\mathrm{AV}$, luego la continuación, la FR con $S$. vulgare inoculado con $B$. cereus y B.cepacia. En la fase I durante la BR del suelo con 75000 ppm de ARA se aplicó la doble y secuencial BS: primero con una SM con la siguiente composición química $(\mathrm{g} / \mathrm{L}): \mathrm{NH}_{4} \mathrm{NO}_{3}$ $10 ; \mathrm{P}_{2} \mathrm{O}_{5} 5 ; \mathrm{K}_{2} \mathrm{O} 5 ; \mathrm{MgO} 0.5 ; \mathrm{S} 0.8 ;$ y $1.0 \mathrm{ml} / \mathrm{L}$ de una solución de microelementos $(\mathrm{g} / \mathrm{L}): \mathrm{H}_{3} \mathrm{BO}_{3}$ 2.86; $\quad \mathrm{ZnSO} 4 \cdot 7 \mathrm{H}_{2} \mathrm{O} \quad 0.22 ; \quad \mathrm{MgCl}_{2} \bullet 7 \mathrm{H} 2 \mathrm{O} \quad 1.81$ (Vallejo et al. 2005, García-Hernández et al. 2007) ajustada a pH 6.8, aplicada c/3dias por un mes (Maldonado-Chávez et al. 2010); al terminar esta fase se cuantificó la concentración del ARA por Soxhlet (Hernández-Valencia \& Mager 2003).

Tabla 1 Diseño experimental para la biorremediación de suelo contaminado con 75000 ppm de aceite residual automotriz por doble y secuencial bioestimulación (fase 1) y posterior fitorremediación (fase 2) con Sorhgum vulgare inoculado con Bacillus cereus y Burkholderia cepacia

\begin{tabular}{|c|c|c|c|c|}
\hline Tratamiento/Sorghum Vulgare & Aceite residual automotriz & Solución mineral & Phaseolus vulgaris (abono verde) & Fitorremediación \\
\hline${ }^{\mathrm{a}}$ Suelo y agua (control absoluto) & - & - & - & - \\
\hline Suelo con aceite residual automotriz (control negativo) & + & - & - & - \\
\hline${ }^{\text {a }}$ Suelo/P. vulgaris $/^{b} S$. vulgare/B. cereus & + & $\mathbf{5 0 \%}$ & + & + \\
\hline${ }^{\text {a }}$ Suelo/P. vulgaris ${ }^{b} /$ S. vulgare/B. cepacia & + & $\mathbf{5 0 \%}$ & + & + \\
\hline${ }^{\text {a }}$ Suelo/P. vulgaris ${ }^{b}{ }^{c} S$. vulgare/B. cereus + B. cepacia & + & $\mathbf{5 0 \%}$ & + & + \\
\hline
\end{tabular}

$(+)=$ agregado $(-)=$ noagregado, $\mathrm{n}=6, \mathrm{a}=$ =tapa debioestimulación/solución mineral, b=bioestimulación/abono verde, c=fitorremediación.

En la segunda BS se inoculo P. vulgaris con los 2 géneros de BPCV indicadas, incorporado como $\mathrm{AV}$, para lo cual B. cereus se hizo crecer en caldo nutritivo $(\mathrm{CN})$ con la siguiente composición (g/L): extracto de carne 3.0; peptona de carne 5.0 y $\mathrm{pH}$ ajustado a 7.0, en tanto que B. cepacia se creció en caldo Pseudomonas cepacia ácido azaleico y triptamina (CPCAT) con la siguiente composición (g/L): triptamina 0.2 ; ácido azaleico 2.0; $\mathrm{K}_{2} \mathrm{HPO}_{4} \quad 4.0 ; \quad \mathrm{KH}_{2} \mathrm{PO}_{4} \quad 4.0 ;$ extracto de levadura $0.02 ; \mathrm{MgSO}_{4} 0.2$ y $\mathrm{pH}$ ajustado a 6.7 ambas BPCV se incubaron a $30^{\circ} \mathrm{C} / 72 \mathrm{~h}$ (Sánchez-Yañez 2007). Entonces por cada 20 semillas de $P$. vulgaris se trataron con $1.0 \mathrm{~mL}$ de cada género de BPCV la concentración se ajustó a la solución patrón No. 0.5 del nefelómetro de McFarlard equivalente a una concentración celular de $1.5 \times 10^{8}$ bacterias $/ \mathrm{mL}$. Comúnmente las semillas $P$. vulgaris o cualquier otra semilla se inoculan con un solo tipo de BPCV para acelerar y mejorar la germinación de sus semillas y acortar los días a emergencia se recomienda usar dos o más géneros de BPCV, por ello en esta parte de la investigación se inocularon $B$. cereus y B. cepacia en relación 1:1 (v/v) de cada una a partir del CN y CPCAT respectivamente (Sánchez-Yáñez 2007). Entonces P. vuglaris tratado con las BPCV se sembró y dejó crecer hasta el estadío fisiológico de plántula equivalente a 25 días después de su siembra, se 
barbecho e incorporó al suelo y se dio un tiempo de mineralización de 30 días (Osaigbovo et al. 2013). Al finalizar este periodo se midió la concentración de ARA por Soxhlet. Para continuar en con la fase II de reducción del ARA remanente, a un nivel no toxico para el suelo (según con la NOM-138 SEMARNAT/SS-2003), mediante la FR con $S$. vulgare inoculado con $B$. cereus y $B$. cepacia de acuerdo al procedimiento previamente descrito para $P$. vulgaris (SánchezYáñez, 2007). Cuando S. vulgare alcanzo su floración en el suelo adherido a sus raíces, cuantificó la concentración del ARA (Domínguez-Rosado et al. 2004). Los datos experimentales fueron sometidos un análisis de varianza (ANOVA), en conjunto con la prueba comparativa de medias Tukey HSD $\mathrm{P}<0.05 \%$ (Ferrera-Cerrato et al. 2006) con el programa estadístico Statgraphics Centurion.

\section{Resultados}

En la Tabla 2 se muestra la BR del suelo con 75000 ppm de ARA por la primera BS con SM/30 días que lo decreció hasta 37500 ppm; en el mismo suelo contaminado con ARA sin biorremediar o control negativo $(\mathrm{CN})$; la atenuación natural no cambio su concentración.

Tabla 2 Biorremediación en un suelo contaminado con 75000 ppm de aceite residual automotriz por bioestimulación una solución mineral por 30 días

\begin{tabular}{ccc}
\hline & \multicolumn{1}{c}{$\begin{array}{c}\text { Aceite residual automotriz } \\
\text { remanente (ppm) }\end{array}$} \\
\cline { 2 - 3 } Tratamiento & $\begin{array}{c}\text { Final } \\
\text { bioestimulación/ } \\
\text { solución mineral }\end{array}$ \\
Suelo y agua (control absoluto) & $\begin{array}{c}\text { Inicial } \\
\text { Suelo con aceite residual automotriz (control negativo) }\end{array}$ & 0.0 \\
\hline Suelo/aceite residual automotriz/bioestimulación/solución mineral & $75000^{\mathrm{b}}+$ & $75000^{\mathrm{b}}$ \\
\hline
\end{tabular}

$\dagger$ Letras distintas con diferencia estadística significativa al $0.05 \%$ según Tukey; *n=6.

Tabla 3 Biorremediación de suelo contaminado con aceite residual automotriz, por doble y secuencial bioestimulación con solución mineral, seguida de la bioestimulación de Phaseolus vulgaris inoculado con Bacillus cereus y/o Burkholderia cepacia incorporado como abono verde

\begin{tabular}{ccc}
\hline & \multicolumn{2}{c}{$\begin{array}{l}\text { Aceite residual automotriz } \\
\text { remanente (ppm) }\end{array}$} \\
\cline { 2 - 3 } Tratamiento & $\begin{array}{c}\text { Final } \\
\text { bioestimulación/ } \\
\text { solución mineral }\end{array}$ & $\begin{array}{c}\text { Final } \\
\text { bioestimulación/ } \\
P . \text { vulgaris }\end{array}$ \\
Suelo y agua (control absoluto) & 0.0 & 0.0 \\
Suelo con aceite residual automotriz (control & $75000^{\mathrm{b}}+$ & $75000^{\mathrm{d}}$ \\
Suelo/bioestimulación/P. vulgaris/B. & $37500^{\mathrm{a}}$ & $20400^{\mathrm{c}}$ \\
Suelo/bioestimulación/P. vulgaris/B. & $37500^{\mathrm{a}}$ & $20100^{\mathrm{b}}$ \\
\hline Suelo/bioestimulación/P. vulgaris/B. cereus $+B$. & $37500^{\mathrm{a}}$ & $10100^{\mathrm{a}}$ \\
\hline
\end{tabular}


En el suelo bioestimulado con SM/30 días el ARA se redujo a 32500 ppm: Mientras que con la segunda BS con $P$. vulgaris inoculado con los 2 géneros de BPCV e incorporado como AV, el ARA se redujo a 10100 ppm. En el suelo bioestimulado con la SM, el ARA disminuyó a 33000 ppm; con la segunda BS con P. vulgaris tratado con B. cereus e incorporado como AV el ARA decreció a 20400 ppm. En el suelo primero bioestimulado con la SM/30 días el ARA se redujo a $32500 \mathrm{ppm}$ y con la segunda $\mathrm{BS}$ con $P$. vulgaris inoculado solo con $B$. cepacia incorporado como AV, el ARA decreció a 21000 ppm. La Tabla 3 se muestra la sumatoria de la biorremediación del suelo impactado con 75000 ppm de ARA por BS con la SM/30días y con la segunda $\mathrm{BS}$ con $P$. vulgaris tratado solo con $B$. cereus e incorporado como AV para finalmente concluir con la FR del ARA restante con $S$. vulgare coinoculado con B. cereus/B. cepacia, cuando la gramínea alcanzo su estadio fisiológico de plántula, en el suelo adherido a sus raíces se detectó que el ARA decreció desde 10100 ppm hasta $2500 \mathrm{ppm}$.

Tabla 4 Biorremediación de suelo contaminado con 75000 ppm de aceite residual automotriz por doble y secuencial bioestimulación con solución mineral y Phaseolus vulgaris inoculado con las BPCV como abono verde y su posterior fitorremediación para minimizar el remanente con Sorghum vulgare inoculado con Bacillus cereus y/o Burkholderia cepacia

\begin{tabular}{ccc}
\hline & \multicolumn{2}{c}{ Aceite residual automotriz remanente (ppm) } \\
\cline { 2 - 3 } Tratamiento & $\begin{array}{c}\text { Final bioestimulación/suma } \\
\text { solución mineral y P. vulgaris }\end{array}$ & $\begin{array}{c}\text { Fitorremediación } \\
\text { S. vulgare } \\
\text { (plántula) }\end{array}$ \\
Suelo y agua (control absoluto) & 0.0 & 0.0 \\
Suelo con aceite residual & $75000^{\mathrm{d}} \dagger$ & $75000^{\mathrm{d}}$ \\
automotriz (control & $20400^{\mathrm{b}}$ & $10300^{\mathrm{b}}$ \\
S. vulgare/B. cereus & $21000^{\mathrm{c}}$ & $16400^{\mathrm{c}}$ \\
\hline S. vulgare/B. cepacia & $10100^{\mathrm{a}}$ & $2500^{\mathrm{a}}$ \\
\hline S. vulgare/B. cereus $+B$. cepacia & & \\
\hline
\end{tabular}

En el suelo fitorremediado con $S$. vulgare (en el mismo estadio fisiológico) tratado solo con $B$. cereus, el ARA se redujo desde 20400 ppm hasta 10300 ppm. En el suelo bioestimulado primero con la SM/30 días el ARA disminuyó a 21000 ppm, con la segunda BS mediante $P$. vulgaris inoculado con B. cepacia e incorporado como AV el ARA disminuyo hasta 16400 ppm. En la Tabla 4 se presenta la sumatoria de la biorremediación de suelo contaminado con 75000 ppm de ARA por BS/30dias con una SM y la segunda y secuencial BS con P. vulgaris tratado con los 2 géneros de BPCV e incorporado como AV y continuar con la FR para minimizar el ARA remanente con $S$. vulgare tratado con B. cereus, cuando esta gramínea alcanzo el nivel de floración, el ARA decreció de 10300 ppm a 5700 ppm. En el suelo contaminado con ARA, BS primero con la $\mathrm{SM} / 30$ días, luego con la siguiente BS con $P$. vulgaris tratado con $B$. cepacia incorporado como AV, con ello el ARA se redujo a $16400 \mathrm{ppm}$. Luego con la FR empleando $S$. vulgare tratado con $B$. cepacia (a nivel de 
floración) la concentración del ARA disminuyo $8300 \mathrm{ppm}$. En el suelo contaminado con ARA, bioestimulado con primero con la SM/30 días, y la siguiente $\mathrm{BS}$ con $P$. vulgaris tratado con ambos géneros de $\mathrm{BPCV}$, incorporado como $\mathrm{AV}$, y posteriormente fitorremediado con $S$. vulgare coinoculado con B. cereus y B. cepacia, ahí ARA disminuyo desde $2500 \mathrm{ppm}$ a $800 \mathrm{ppm}$. (Tabla 5)

Tabla 5 Fitorremediación del suelo contaminado con aceite residual automotriz remante con Sorghum vulgare a floración, inoculado con Bacillus cereus y/o Burkholderia cepacia, posterior a su bioestimulación con solución mineral y Phaseolus vulgaris inoculado con las BPCV como abono verde

\begin{tabular}{ccc}
\hline & \multicolumn{2}{c}{ Aceite residual automotriz remanente (ppm) } \\
\cline { 2 - 3 } Tratamiento & Fitorremediació & Fitorremediación \\
& $\mathrm{n}$ S. vulgare & S. vulgare (floración) \\
Suelo y agua (control absoluto) & 0.0 & 0.0 \\
Suelo con aceite residual automotriz & & $75000^{\mathrm{d}}$ \\
(control negativo) & $75000^{\mathrm{d}_{\dagger}}$ & $5700^{\mathrm{b}}$ \\
S. vulgare/B. cereus & $10300^{\mathrm{b}}$ & $8300^{\mathrm{c}}$ \\
\hline S. vulgare/Burkholderia cepacia & $16400^{\mathrm{c}}$ & $800^{\mathrm{a}}$ \\
\hline S. vulgare/B. cereus + B.cepacia* & $2500^{\mathrm{a}}$ & \\
\hline
\end{tabular}

$\dagger$ Letras distintas indican diferencia significativa al $0.05 \%$ según Tukey, $\mathrm{n}^{*}=6$.

\section{Discusión}

La biorremediación del suelo contaminado con 75000 ppm de ARA, mediante la primera BS con $\mathrm{SM} / 30$ días, sugiere que las sales de los elementos esenciales del tipo de: $\mathrm{NH}_{4} \mathrm{NO}_{3}, \mathrm{P}_{2} \mathrm{O}_{5}$, $\mathrm{K}_{2} \mathrm{O}$ y $\mathrm{MgO}$ equilibraron el desbalance que inhibe la actividad de la microbiota heterotrófica aerobia nativa oxidante de los HC del ARA (Vallejo et al. 2005, Gómez et al. 2009). Al respecto se reporta que en suelo bioestimulado impactado con mezclas de $\mathrm{HC}$ alifáticos como los del ARA, existen microorganismos nativos heterotróficos aerobios que los oxidan durante los primeros 30 días, al ser la fracción más simple de mineralizar (Abdulsalam \& Omale 2009). En el suelo con 75000 ppm de ARA sin bioestimular o control negativo, la atenuación natural no cambio ésta elevada concentración del ARA que causa la inhibición prácticamente total de la actividad microbiana aerobia heterotrófica del suelo (Asquith et al. 2012). En la BR del suelo contaminado con 75000ppm del ARA, primero por BS con la SM/30 días y luego con segunda BS con $P$. vulgaris tratado con ambos géneros de la $\mathrm{BPCV}$, que se incorporó como $\mathrm{AV}$, sugiere que este aumento la concentración y disponibilidad de Nitrógeno $(\mathrm{N})$ orgánico y mineral para reestablecer el balance de nutrientes e indujo una mayor actividad microbiana heterotrófica aerobia de oxidación de los HC del ARA (Diab 2008, Sangabriel et al. 2006). Similar a lo observado en el suelo contaminado con 75000ppm de ARA bioestimulado por principio con la SM/30 y luego con la segunda BS mediante $P$. vulgaris tratado solo con B. cereus incorporado como AV, ambas acciones facilitaron y aceleraron la reducción del ARA, en comparación con lo registrado cuando el suelo impactado con ARA se bioestimuló primero con la SM/30 días y luego con la segunda BS mediante $P$. vulgaris inoculado con $B$. cepacia que se incorporó como AV. En contraste con el 
suelo impactado con 75000 ppm de ARA bioestimulado con la $\mathrm{SM} / 30$ días y posterior segunda BS con $P$. vulgaris tratado con ambos géneros de BPCV, incorporado como AV; aquí la doble y secuencial $\mathrm{BS}$ potenció la rápida mineralización del ARA: un aspecto innovador para la biorrestauración del suelo contaminado con una relativamente alta concentración de ARA (Hernández-Bejarano et al. 2011, Asquith et al. 2012).

Los resultados anteriores apoyan que en el suelo existen microorganismos heterotróficos aerobios autóctonos capaces oxidar el ARA (datos no mostrados), incapaces de actuar cuando la concentración del ARA es relativamente elevada, condición ambiental agravada por la deficiencia de minerales esenciales. En contraste esta microbiota nativa fue bioestimulada con componentes de la SM y con la segunda bioestimulación derivada de los compuestos orgánicos de $\mathrm{N}$ producto de la descomposición de $P$. vulgaris al incorporarse suelo como $\mathrm{AV}$; ambos acciones favorecieron una relativamente rápida y eficaz mineralización del ARA (Pardo et al. 2004, Ogbo 2009). En el suelo impactado con 75000 ppm de ARA, bioestimulado primero con la SM/30 días y luego con la segunda BS mediante $P$. vulgaris trata et al do con los 2 géneros de $\mathrm{BPCV}$, incorporada como $\mathrm{AV}$, se logró reducir drásticamente la concentración del ARA. Luego con la FR usando $S$. vulgare inoculado con un o los dos géneros de BPCV, a nivel de la rizósfera de la gramínea se mejoró su capacidad para fitodegradar el ARA. Al respecto se ha reportado que en suelo contaminado con HC del petróleo, la aplicación de la FR es posible porque S. vulgare, posee un sistema radical que facilita la degradación del ARA (Banks et al.
2002, Ferrera-Cerrato et al. 2006). Con estos resultados se demuestra que en el suelo contaminado con una elevada concentración ARA es posible una máxima disminución de su concentración, con $S$. vulgare que al liberar exudados radicales estimulo la actividad oxidante de $\mathrm{HC}$ de B. cereus, de B. cepacia y de otros microorganismos nativos del suelo para mineralizar el ARA a un nivel no tóxico para la vida (Ferrera-Cerrato et al. 2008, Abdulsalam \& Omale 2009, Maldonado-Chávez et al. 2010, Hernández-Bejarano et al. 2011). Los resultados anteriores también refuerzan la hipótesis de que la BR de suelo contaminado con una concentración relativamente alta de ARA, requiere una doble y secuencial $\mathrm{BR}$, seguida de la FR con una planta tolerante a la fitoxicidad del ARA, potenciada con géneros de BPCV. Con una evidente disminución del ARA a un nivel no tóxico para el ambiente, en un tiempo relativamente corto, que no es posible alcanzar con cualesquier de ambas estrategias es aplicado de manera individual

\section{Conflictos de intereses}

Los autores declaran que no existen conflictos de interés.

\section{Agradecimientos}

Al proyecto 2.7 (2015) apoyado por la Coordinación Investigación Científica-UMSNH y BIONUTRA S.A de CV, Maravatio, Mich, México y CONACYT-México por beca-tesis para el primer autor. 


\section{Literatura citada}

Bentancor A. El rol epidemiologico de las mascotas en el ciclo de transmisión urbana de cepas STEC. Medicina (Buenos Aires) 2006; 66 (Supl III): $37-41$.

Abdulsalam S, Omale A. Comparison of Biostimulation and Bioaugmentation Techniques for the Remediation of Used Motor Oil Contaminated Soil. Braz Arch Biol Techno. 2009. 52:747-754.

Agamuthu P, Abioye OP, Abdul A. Phytoremediation of soil contaminated with used lubricating oil using Jatropha curcas L. J Hazard Mater. 2010. 179:891-894.

Asquith E, Geary M, Nolan A, Evans C. Comparative Bioremediation of Petroleum Hydrocarbon-Contaminated Soil by Biostimulation, Bioaugmentation and Surfactant Addition . J Environ Sci Eng. A.1. 2012. 1: 637650.

Banks MK, Kulakow P, Schwab AP, Chen Z, Rathbone K. Degradation of Crude Oil in the Rhizosphere of Sorghum bicolor. Int J Phytorem. 2002. 5: 225-234.

Basumatary B, Bordoloi S, Sarma HP. Crude OilContaminated Soil Phytoremediation by Using Cyperus brevifolius (Rottb.) Hassk. Water Air Soli Poll. 2012a; 223(6): 3373-3383.

Diab EA. Phytoremediation of Oil Contaminated Desert Soil Using the Rhizosphere Effects. Global J Environ Res. 2008; 2: 66-73.

Dominguez-Rosado E, Pichtel J. Phytoremediation of Soil Contaminated with Used Motor Oil: II. Enhanced Microbial Activities from Laboratory and Growth Chamber Studies. Environ Eng Sci. 2004; 21: 169-180.
Dominguez-Rosado E, Pichtel J, Coughlin $\mathrm{M}$. Phytoremediation of Soil Contaminated with Used Motor Oil: I. Enhanced Microbial Activities from Laboratory and Growth Chamber Studies. Environ Eng Sci. 2004; 21,157-169.

Ferrera-Cerrato R, Alarcón A, Trejo-Aguilar D, Sangabriel W, Mendoza-López M, CruzSánchez J, et al. Fitorremediación de un suelo contaminado con combustóleo utilizando Phaseolus coccineus y fertilización orgánica e inorgánica. Agrociencia. 2008. 41: 817-826.

Ferrera-Cerrato R, Rojas-Avelizapa NG, PoggiVaraldo HM, Alarcón A, Cañizares-Villanueva RO. Procesos de biorremediación de suelo y agua contaminados por hidrocarburos del petróleo y otros compuestos orgánicos. Rev Latinoam Microbiol. 2006.48, 179-187.

García-Hernández D, Sosa-Aguirre CR, SánchezYáñez JM. Biorremediación de agua doméstica contaminada con aceite residual automotriz por bioestimulación. Ing Hidraul Mex. 2007. 22:113118.

Gómez W, Gaviria J, Cardona S. Evaluación de la bioestimulación frente a la atenuación natural y la bioaumentación en un suelo contaminado con una mezcla de gasolina-diesel. Dyna. 2009. 76: 83-93.

Hernández-Bejarano A, Rodríguez A, Barrios M, Rodríguez A, Guerrero A. Análisis de la diversidad microbiana de la rizósfera de tres especies vegetales de un suelo contaminado con hidrocarburos del petróleo y su potencial rizoremediador. Rev Latinoam Biotecnol Amb Algal. 2011. 2: 1-17.

Hernández-Valencia L, Mager D. Uso de Panicum maximun y Brachiaria brizantha para fitorremediar suelos contaminados con un 
crudo de petróleo liviano. Bioagro. 2003.15: 149-155.

Maldonado-Chávez E, Rivera-Cruz M, IzquierdoReyes F, Palma-López D. Efectos de rizósfera, microorganismos y fertilización en la biorremediación y fitorremediación de suelos con petróleos crudo nuevo e intemperizado. Universidad y Ciencia. 2010. 26: 121-136.

Ogbo EM. Effects of diesel fuel contamination on seed germination of four crop plants Arachis hypogaea, Vigna unguiculata, Sorghum bicolor and Zea mays. Afr J Biotechnol. 2009. 8(2): 250-253.

Ogbo EM, Ufuoma A, Gloria O. Screening of four common Nigerian weeds for use in phytoremediation of soil contaminated with spent lubricating oil. Afri J Plant Sci. 2009; 3(5):102-106.

Osaigbovo A, Law-Ogbomo K, Agele S. Effects of spent engine oil polluted soil and organic amendment on soil chemical properties, micro-flora on growth and herbage of Telfairia occidentalis (Hook F). J Pure Appl Sci. 2013. 6: 72-78.
Pardo JL, Perdomo CM, Benavides LJ. Efecto de la adición de fertilizantes inorgánicos compuestos en la degradación de hidrocarburos en suelo contaminados con petróleo. NOVA. 2004. 2: 40-49.

Sánchez-Yáñez JM. Breve Tratado de Microbiología Agrícola, Teoría y Práctica. Ed. Universidad Michoacana de San Nicolás de Hidalgo, CIDEM, Secretaria de Desarrollo Agropecuario del Gobierno del Estado de Michoacan. CONSUTENTA, SA de CV Morelia, Mich, México. 2007. ISBN: 978970-95424-1-7.

Sangabriel W, Ferrera-Cerrato R, Trejo-Aguilar D, Mendoza-López MR, Cruz-Sánchez J, López-Ortiz C, et al. Tolerancia y fitorremediación de suelo contaminado con combustóleo por seis especies vegetales. Rev Int Contam Ambient. 2006. 22: 63-73.

Vallejo V, Salgado L, Roldan F. Evaluación de la bioestimulación en la biodegradación de TPH en suelos contaminados con petróleo. Rev Colomb Biotecnol. 2005. 2:67-78.

Zand AD, Bidhendi GN, Mehrdadi N. Phytoremediation of total petroleum hydrocarbons (TPHs) using plant species in Iran. Turk J Agric For. 2010. 34: 429-438. 